ORIGINAL ARTICLE

\title{
Severity of the ductal shunt: a comparison of different markers
}

\author{
M El Hajiar, G Vaksmann, T Rakza, G Kongolo, L Storme
}

Arch Dis Child Fetal Neonatal Ed 2005;90:F419-F422. doi: 10.1136/adc.2003.027698

See end of article for authors' affiliations .....................

Correspondence to: Dr Storme, Service de Médecine Néonatale, Hôpital Jeanne de Flandre, CHRU de Lille, Lille cédex 59037, France Istorme@chru-lille.fr

Accepted 16 March 2005
Background: When the ductus arteriosus (DA) is patent, the ductal shunt is proportional to the ratio of left ventricular output (LVO) to systemic blood flow. Systemic blood flow can be estimated by measuring flow in the superior vena cava (SVC).

Objective: To re-evaluate the accuracy of standard echocardiographic markers of patent ductus arteriosus (PDA) using LVO/SVC flow ratio.

Methods: Prospective study. Preterm infants of 24-30 weeks gestational age and postnatal age less than 48 hours. The following echocardiographic criteria were measured: left atrial to aortic root ratio (LA/Ao); DA diameter by B mode and colour Doppler; mean and end diastolic flow velocity of the left pulmonary artery (LPA); LVO; SVC flow.

Results: Twenty three preterm infants were enrolled (median gestational age 28 weeks (range 24-30), median birth weight $840 \mathrm{~g}$ (500-1440)). The DA was closed in eight (mean (SD) LVO/SVC 2.4 (0.3)) and open in 15 (mean (SD) LVO/SVC 4.5 (0.6)). An LA/Ao ratio $\geqslant 1.4$, a DA diameter $\geqslant 1.4 \mathrm{~mm} / \mathrm{kg}$, and a mean and end diastolic flow velocity of LPA respectively $\geqslant 0.42$ and $\geqslant 0.20 \mathrm{~m} / \mathrm{s}$ identified an LVO/SVC $\geqslant 4$ with a sensitivity and a specificity above $90 \%$.

Conclusion: This study indicates that LA/Ao ratio, DA diameter, and mean and end diastolic flow velocity of the LPA are accurate markers of PDA. These standard echocardiographic variables are easy to measure and need less skill and resources than direct measurements of ductal shunt.
T he incidence of patent ductus arteriosus (PDA) in preterm infants varies from $20 \%$ to $60 \%$, ${ }^{1}$ depending on the diagnostic criteria used and the population studied. ${ }^{2}$ Significant left to right shunting as a result of PDA in premature infants contributes to increased morbidity such as intraventricular haemorrhage, necrotising enterocolitis, kidney injury, heart failure, and chronic lung disease. ${ }^{3-7}$ The mechanisms include decreased peripheral organ perfusion and increased pulmonary perfusion. ${ }^{8-11}$

Assessment of the significance of the ductal flow remains difficult. Clinical symptoms, electrocardiography, and chest radiographic anomalies have been found to be neither accurate nor specific. ${ }^{12} 13$ Doppler echocardiography proved better than clinical examination in grading PDA. ${ }^{14}{ }^{15}$

Echocardiographic criteria such as left atrial/aortic root ratio $(\mathrm{LA} / \mathrm{Ao}) \geqslant 1.4{ }^{1}$ ductus arteriosus (DA) diameter $\geqslant 1.5 \mathrm{~mm},{ }^{16}{ }^{17}$ end diastolic flow velocity in the left pulmonary artery $\left(\right.$ LPA) $\geqslant 0.2 \mathrm{~m} / \mathrm{s},{ }^{18}$ increased left ventricular output (LVO), ${ }^{11} 1920$ and increased peripheral vascular resistance index ${ }^{7}$ have been associated with significant ductal shunting. In some of these studies, clinical or radiographic signs or other indirect echocardiographic variables were used as the gold standard methods. ${ }^{161721}$ However, it is not known which is the best non-invasive method for evaluating the magnitude of the left to right shunting through the DA.

Evans et $a^{22}$ showed that systemic blood flow can be estimated by measuring superior vena cava (SVC) flow. In 25 uncomplicated infants born before 30 weeks with a closed DA, mean (SD) SVC flow to LVO ratio (SVC/LVO) was 37 (11)\%; median intraobserver and interobserver variability were $8.1 \%$ and $14 \%$ respectively. ${ }^{22}$ Therefore an estimate of total systemic blood flow can be derived from SVC flow multiplied by 2.7. When the duct is patent, LVO represents the sum of systemic blood flow and the ductal shunt. ${ }^{411}$ The ductal flow can be calculated as LVO $-2.7 \times$ SVC flow. The equation can be rewritten as follows:

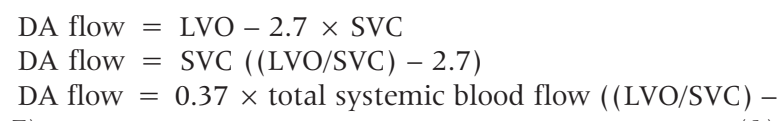

According to equation 1 , the ductal shunt is proportional to the $\mathrm{LVO} / \mathrm{SVC}$ ratio.

The aim of our study was to re-examine the accuracy of the main standard markers of PDA using the LVO/SVC ratio as the yardstick.

\section{PATIENTS AND METHODS}

We conducted a prospective study in the neonatal intensive care unit of the University Hospital of Lille between June 2000 and May 2001. The entry criteria were preterm birth at 24-30 weeks gestational age, a postnatal age less than 48 hours, and informed parental consent. Newborn infants who had congenital heart disease or been treated with high frequency oscillatory ventilation were excluded from the study. ${ }^{23}$ Echocardiographic data were collected using a Vingmed FMC 750 echocardiographic system. Measurements were performed by the same investigator. Averages of three to five consecutive readings for the vessel diameter and flow velocity integrals were used. Angle of insonation was less than $20^{\circ}$. The following Doppler echocardiographic variables were measured:

- LA/Ao ratio: $M$ mode pictures of the LA and Ao were obtained from a parasternal long axis view ${ }^{1}$

- Internal diameter of the DA by both B mode and colour Doppler from the high left parasternal view. ${ }^{16}$ The DA diameter was related to the infant's weight and expressed as $\mathrm{mm} / \mathrm{kg}$

Abbreviations: DA, ductus arteriosus; LA/Ao, left atrial/aortic root ratio; LPA, left pulmonary artery; LVO, left ventricular output; PDA, patent ductus arteriosus; ROC, receiver operating characteristics; SVC,
superior vena cava 


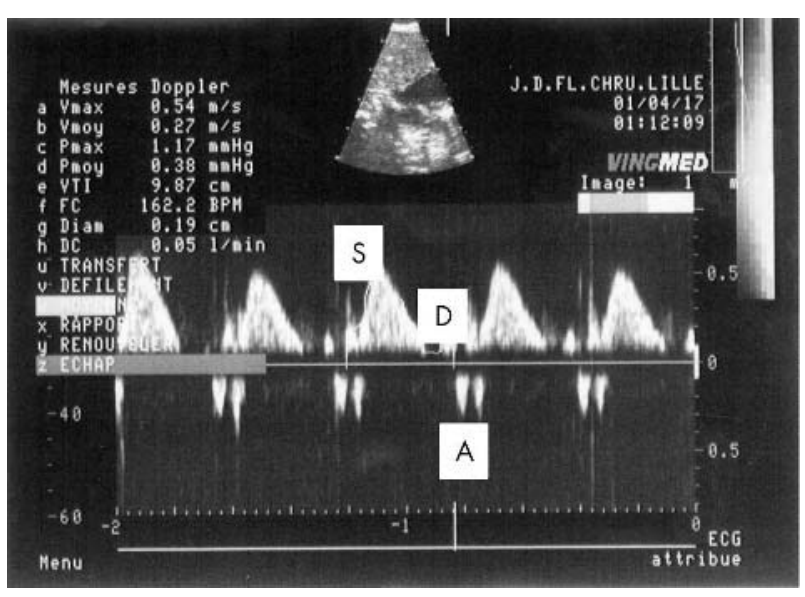

Figure 1 The superior vena cava flow obtained by low subcostal view, with the diastolic (D), systolic (S), and atrial (A) waves.

- Mean and end diastolic flow velocity of the LPA was measured with pulsed Doppler using a high left parasternal view ${ }^{18} 24$

- LVO: diameter of the LVO tract was measured from a parasternal long axis view using the leading edge technique. The flow velocity time integral was measured from the apical view with the sample volume placed in the LVO tract. ${ }^{25}$ The heart rate was measured from peak to peak intervals of the Doppler velocity time signals

- SVC flow was measured as previously described..$^{22}$ The flow was imaged from a low subcostal view. The Doppler sample volume was placed at the junction of the SVC and right atrium. The flow consists of three waves: systolic, diastolic, and atrial (fig 1). The diameter of the SVC was imaged by $\mathrm{M}$ mode, from the right or left parasternal long axis view, at the junction of the SVC and right atrium. Because of the variation in vessel diameter throughout the cardiac cycle, a mean of the maximum and minimum diameter during the cardiac cycle was used for calculation of the flow (fig 2).

\section{Statistical analysis}

Data were analysed with a personal computer based statistics package (SPSS for Windows). Standard simple linear regression analysis was used to assess the relation between $\mathrm{LVO} / \mathrm{SVC}$ ratio and LA/Ao ratio, diameter of the DA, and

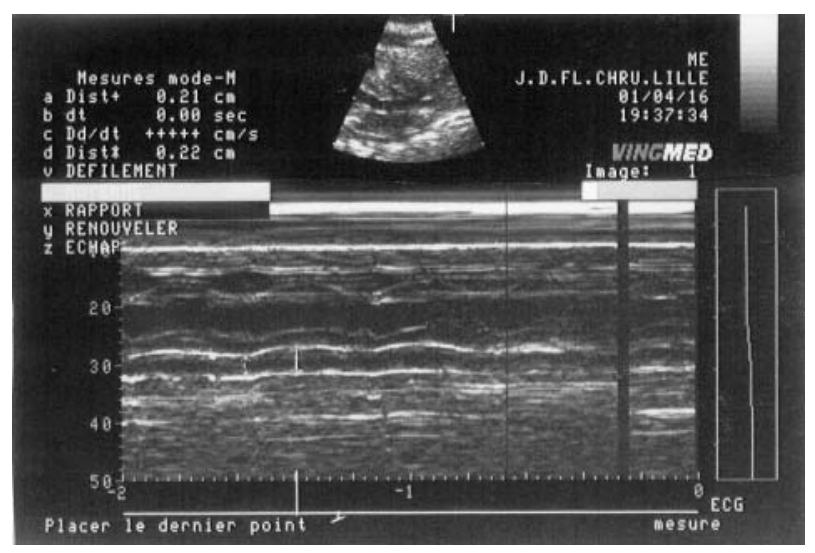

Figure 2 The superior vena cava diameter obtained by $M$ mode in left parasternal long axis view. The maximum and minimum diameters were measured. mean and end diastolic flow velocity of the LPA. Sensitivity and specificity were computed for each variable to determine which level best predicted substantial patency of a DA. A $\mathrm{LVO} / \mathrm{SVC}$ ratio $\geqslant 4$ was considered to be related to PDA as the DA flow represents more than $50 \%$ of the total systemic blood flow (according to equation 1 ).

Receiver operating characteristics (ROC) curves were obtained using $\mathrm{LVO} / \mathrm{SVC}$ ratio $\geqslant 4$ as the actual state variable and LA/Ao, DA diameters, or mean and end diastolic flow velocity of the LPA as test result variables. Area under the ROC curves and best cut-off values for each variable were calculated. $\mathrm{p}<0.05$ was considered significant.

\section{RESULTS}

During the study period, 23 preterm infants were included (10 male). Gestational ages ranged from 24 to 30 weeks (median 28). Birth weights ranged from 500 to $1440 \mathrm{~g}$ (median $840 \mathrm{~g}$ ).

LPA flow analysis could not be performed in two infants with LPA stenosis. ${ }^{26}$ The DA was closed in eight infants (35\%; mean (SD) LVO/SVC $2.4(0.3))$ and open in 15 infants (65\%; mean LVO/SVC $4.5(0.6))$.

The LA/Ao ratio correlated significantly with LVO/SVC $(\mathrm{p}<0.01$; fig $3 \mathrm{~A})$, and the optimal cut-off point was 1.4 to predict PDA defined by a LVO/SVC ratio $\geqslant 4$, with a sensitivity of $92 \%$ and a specificity of $91 \%$. The area under the ROC curve was 0.94. DA diameters (in B mode or colour Doppler) expressed as infant's weight correlated with LVO/SVC $(\mathrm{p}<0.01$; fig $3 \mathrm{~B}, \mathrm{C})$. The best cut-off point was $1.4 \mathrm{~mm} / \mathrm{kg}$ in both B mode and colour Doppler, with a sensitivity of $94 \%$ and a specificity of $90 \%$. The area under the ROC curve was 0.89 in B mode and 0.92 in colour Doppler mode. Mean and end diastolic flow velocities of the LPA both correlated with LVO/SVC $(p<0.02$ and $p<0.01$ respectively; fig $3 D, E)$. The optimal cut-off points were respectively $0.42 \mathrm{~m} / \mathrm{s}$ (sensitivity $91 \%$, specificity $92 \%$ ) and $0.20 \mathrm{~m} / \mathrm{s}$ (sensitivity $92 \%$, specificity $100 \%$ ). The areas under the ROC curves were respectively 0.90 and 0.88 .

\section{DISCUSSION}

The accuracy of the usual echocardiographic markers of PDA was re-evaluated using the LVO/SVC flow ratio as the gold standard. We found that LA/Ao ratio, ductal diameter in both B mode and colour Doppler, mean flow velocity of the LPA, and end diastolic flow velocity of the LPA correlated significantly with the LVO/SVC ratio. Furthermore, an LA/ Ao ratio $\geqslant 1.4$, a ductal diameter (B mode and colour Doppler) $\geqslant 1.4 \mathrm{~mm} / \mathrm{kg}$, a mean flow velocity of the LPA $\geqslant 0.42 \mathrm{~m} / \mathrm{s}$, and an end diastolic flow velocity of the LPA $\geqslant 0.20 \mathrm{~m} / \mathrm{s}$ identified an $\mathrm{LVO} / \mathrm{SVC}$ ratio $\geqslant 4$ with a sensitivity and specificity above $90 \%$.

We defined the LVO/SVC ratio as the ideal criterion for evaluating the flow of the ductal shunt for four reasons: (a) according to equation 1 , the flow of the ductal shunt is proportional to the LVO/SVC ratio; $(b)$ the $\mathrm{LVO} / \mathrm{SVC}$ ratio is a sensitive marker of ductal flow because LVO increases ${ }^{77}$ and SVC decreases ${ }^{28}$ when ductal shunt increases; (c) the LVO/ SVC ratio is independent of interatrial shunting through the permeable foramen ovale ${ }^{29} ;(d)$ the LVO/SVC ratio is easy to calculate and simple to conceptualise-indeed, it represents the actual component of the left cardiac output that perfuses the upper body. As about $80 \%$ of upper blood flow goes to the brain, the LVO/SVC ratio may also be a useful marker of cerebral perfusion. However, the precise threshold of ductal shunting above which complications may occur is not known. In our study, we chose a ratio $\geqslant 4$ to define a haemodynamically significant ductal shunt. According to equation 1, the volume of the ductal shunt is equal to half of the total systemic blood flow when the LVO/SVC ratio is 4 . A 

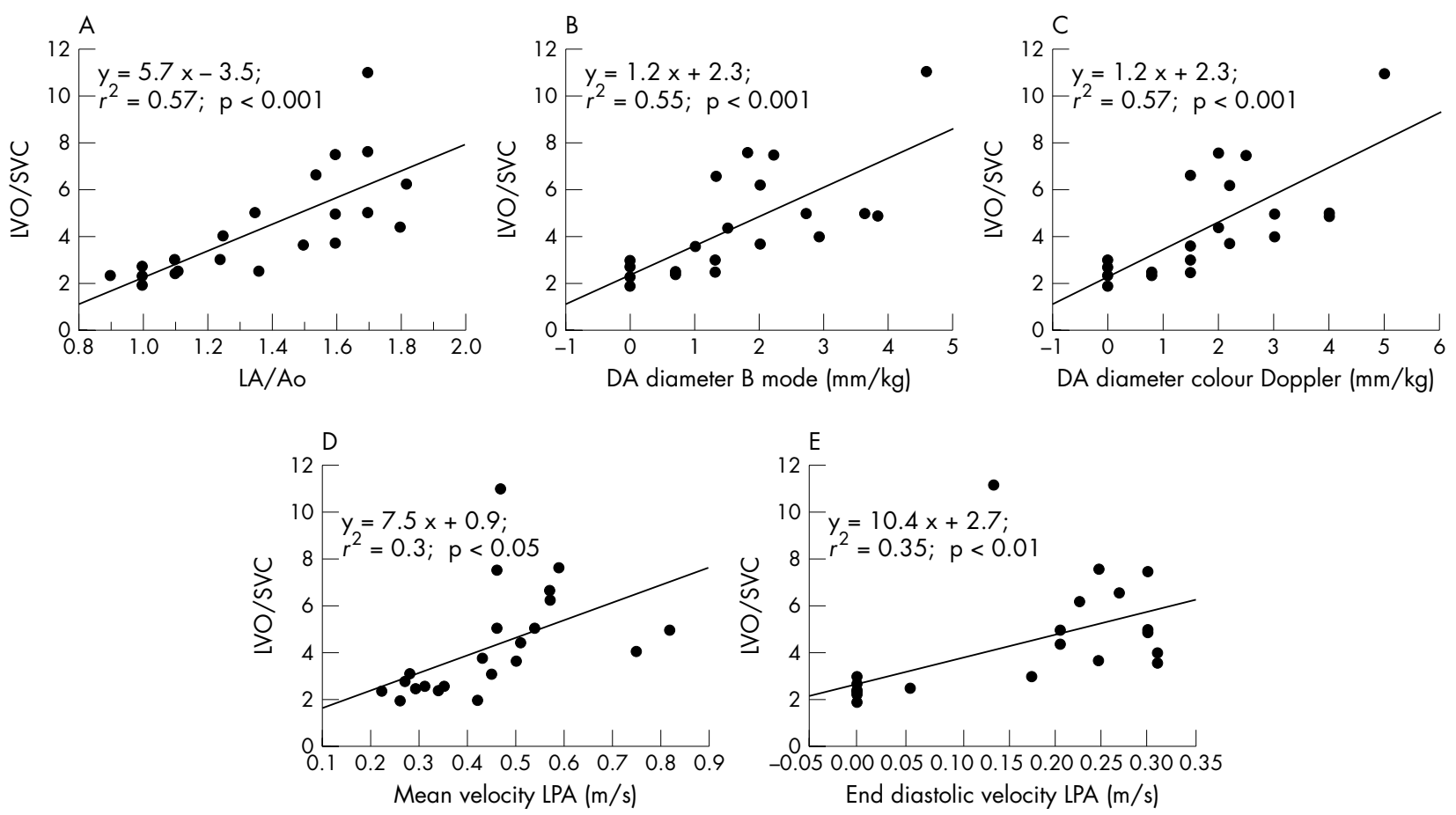

Figure 3 Standard linear regression analysis for comparison between (A) left atrial to aortic root ratio (LA/Ao) and left ventricular output/superior vena cava flow (LVO/SVC) ratio, (B) diameter of the ductus arteriosus (DA) in B mode and LVO/SVC ratio, (C) diameter of the DA in colour Doppler and LVO/SVC ratio, (D) mean blood flow velocity of the left pulmonary artery (LPA) and LVO/SVC ratio, and (E) end diastolic blood flow velocity of the LPA and LVO/SVC ratio.

previous study suggested that an LVO/right ventricular output ratio close to 2 is associated with increased intraventricular haemorrhage and periventricular leucomalacia. ${ }^{27} \mathrm{~A} \mathrm{LVO/right} \mathrm{ventricular} \mathrm{output} \mathrm{ratio} \mathrm{equal} \mathrm{to} 2$ indicates a ductal shunt volume close to $50 \%$ of the LVO in the absence of significant atrial shunting. Thus, evidence exists that an $\mathrm{LVO} / \mathrm{SVC}$ ratio $\geqslant 4$ may identify significant left to right ductal shunting, which indicates a risk of hypoperfusion in preterm infants.

Previous studies found that an LA/Ao ratio of $1.4^{30}$ or $1.5,{ }^{16}$ a ductal diameter of $1.5 \mathrm{~mm},{ }^{16}{ }^{17}$ and a diastolic flow velocity in the LPA of $0.2 \mathrm{~m} / \mathrm{s}^{18}$ were the optimal cut-off points for identifying PDA. Our results are in accordance with these previous studies. However, we found that a ductal diameter $\geqslant 1.4$ expressed as $\mathrm{mm} / \mathrm{kg}$ provides better sensitivity and specificity than an absolute diameter. This finding seems logical as a $2 \mathrm{~mm}$ DA may not have the same haemodynamic consequence in a $500 \mathrm{~g}$ and a $1400 \mathrm{~g}$ preterm newborn. Moreover, we showed that a mean flow velocity of the LPA $\geqslant 0.42 \mathrm{~m} / \mathrm{s}$ may be useful for identifying significant left to right shunting.

Measurement of SVC flow requires an experienced and skilful operator and considerable training and practice in Doppler echocardiography. Thus, although LVO/SVC is clearly useful in evaluating the degree of DA shunting, this direct Doppler imaging method cannot be used routinely in neonatal intensive care units. This study indicates that

\section{What is already known on this topic}

- Previous studies reported that an LA/Ao ratio $\geqslant 1.4$ or 1.5, a ductal diameter $\geqslant 1.5 \mathrm{~mm}$, and a diastolic flow velocity in the $L P A \geqslant 0.2 \mathrm{~m} / \mathrm{s}$ identify significant left to right shunting through PDA in premature infants
LA/Ao, DA diameter expressed as $\mathrm{mm} / \mathrm{kg}$, and mean and end diastolic flow velocity of the LPA are accurate tools for identifying PDA. These standard echocardiographic variables are easy to measure and need less skill and resources than direct SVC measurements.

The study has some limitations. The small sample size may have resulted in imprecision in the estimates of the cut-off values (including sensitivities and specificities).

\section{CONCLUSIONS}

Evaluation of the volume of left to right shunting through the DA remains challenging. Clinical and radiographic examination are not sufficiently reliable to quantify ductal shunt. Using the LVO/SVC ratio as the yardstick for estimating ductal shunting outflow, our study confirms the accuracy of an LA/Ao ratio $\geqslant 1.4$ and an end diastolic flow velocity of the LPA $\geqslant 0.20 \mathrm{~m} / \mathrm{s}$. To determine the size of the ductal shunt, DA diameter should be expressed as $\mathrm{mm} / \mathrm{kg}$. A DA diameter $\geqslant 1.4 \mathrm{~mm} / \mathrm{kg}$ and a mean flow velocity of the LPA $\geqslant 0.42 \mathrm{~m} / \mathrm{s}$ are also good indicators of patent ductus arteriosus.

\section{What this study adds}

- The ductal shunt is proportional to the LVO/SVC flow ratio. Using the LVO/SVC ratio as the yardstick for estimating ductal shunting oufflow, we re-examined the accuracy of standard markers of PDA

- The study confirms the accuracy of an LA/Ao $\geqslant 1.4$ and an end diastolic flow velocity of the LPA $\geqslant 0.20 \mathrm{~m} / \mathrm{s}$ in identifying significant left to right shunting through PDA, and shows that a DA diameter $\geqslant 1.4 \mathrm{~mm}$ when expressed per $\mathrm{kg}$ and a mean blood flow velocity in the LPA $\geqslant 0.42 \mathrm{~m} / \mathrm{s}$ are also good indicators in premature infants 


\section{Authors' affiliations}

M El Hajiar, T Rakza, L Storme, Clinique de Médecine Néonatale,

Hôpital Jeanne de Flandre, CHRU de Lille, Lille, France

G Vaksmann, Service de Cardiologie Pédiatrique, CHRU de Lille

G Kongolo, Service de Réanimation Pédiatrique, CHRU d'Amiens,

Amiens, France

Competing interests: none declared

\section{REFERENCES}

1 lyer $\mathbf{P}$, Evans N. Re-evaluation of the left atrial to aortic root ratio as a marker of patent ductus arteriosus. Arch Dis Child 1994;70:112-17.

2 Ellison RC, Peckham GJ, Lang P, et al. Evaluation of the preterm infant for patent ductus arteriosus. Pediatrics 1983:71:364-72.

3 Bomelburg T, Jorch G. Abnormal blood flow patterns in renal arteries of small preterm infants with patent ductus arteriosus detected by Doppler ultrasonography. Eur J Pediatr 1989;148:660-4.

4 Kluckow M, Evans N. Ductal shunting, high pulmonary blood flow, and pulmonary hemorrhage. J Pediatr 2000;137:68-72.

5 Shortland DB, Gibson NA, Levene MI, et al. Patent ductus arteriosus and cerebral circulation in preterm infants. Dev Med Child Neurol 1990;32:386-93.

6 Maruyama K, Koizumi T, Tomomasa T, et al. Intestinal blood-flow velocity in uncomplicated preterm infants during the early neonatal period. Pediatr Radiol 1999;29:472-7.

7 Shimada S, Kasai T, Konishi M, et al. Effects of patent ductus arteriosus on left ventricular output and organ blood flows in preterm infants with respiratory distress syndrome treated with surfactant. J Pediatr 1994; 125:270-7.

8 Ichihashi K, Shiraishi H, Endou H, et al. Cerebral and abdominal arterial hemodynamics in preterm infants with patent ductus arteriosus. Acta Paediatr Jpn 1990;32:349-56.

9 Bomelburg T, Jorch G. Abnormal blood flow patterns in renal arteries of small preterm infants with patent ductus arteriosus detected by Doppler ultrasonography. Eur J Pediatr 1989;148:660-4.

10 Alverson DC, Eldridge MW, Johnson JD, et al. Effect of patent ductus arteriosus on left ventricular output in premature infants. Am J Perinato 1984;1:216-22.

11 Linder W, Seidel M, Versmold HT, et al. Stroke volume and left ventricular output in preterm infants with patent ductus arteriosus. Pediatr Res 1990;27:278-81.

12 Evans N. Diagnosis of patent ductus arteriosus in the preterm newborn. Arch Dis Child 1993;68:58-61

13 Kupferschmid C, Lang D, Pohlandt F. Sensitivity, specificity and predictive value of clinical findings, m-mode echocardiography and continuous-wave Doppler sonography in the diagnosis of symptomatic patent ductus arteriosus in preterm infants. Eur J Pediatr 1988; 147:279-82.
14 Skelton R, Evans N, Smythe JA. Blinded comparison of clinical and echocardiographic evaluation of the preterm infant for patent ductus arteriosus. J Paediatr Child Health 1994;30:406-11.

15 Hirsimaki H, Kero P, Wanne O. Doppler ultrasound and clinical evaluation in detection and grading of patent ductus arteriosus in neonates. Crit Care Med 1990;18:490-3.

16 Kluckow M, Evans N. Early echocardiographic prediction of symptomatic patent ductus arteriosus in preterm infants undergoing mechanical ventilation. J Pediatr 1995; 127:774-9.

17 Evans N, lyer P. Assessment of ductus arteriosus shunt in preterm infants supported by mechanical ventilation: effect of interatrial shunting. J Pediatr $1994 ; 125: 778-85$

18 Suzmura H, Nitta A, Tanaka O. Diastolic flow velocity of left pulmonary artery of patent ductus arteriosus in preterm infants. Pediatr Int 2001;43:146-51.

19 Walther FJ, Kim DH, Ebrahimi M, et al. Pulsed Doppler measurement of left ventricular output as early predictor of symptomatic patent ductus arteriosus in very preterm infants. Biol Neonate 1989;56:121-8.

20 Pladys $\mathbf{P}$, Wodey E, Beuchee A, et al. Left ventricle output and mean arterial blood pressure in preterm infants during the 1 st day of life. Eur J Pediatr 1999:158:817-24.

21 Davies MW, Betheras FR, Swaminathan M. A preliminary study of the application of the transductal velocity ratio for assessing persistent ductus arteriosus. Arch Dis Child Fetal Neonatal Ed 2000;82:F195-9.

22 Kluckow M, Evans N. Superior vena cava flow in newborn infants: a novel marker of systemic blood flow. Arch Dis Child Fetal Neonatal Ed 2000;82:F182-7.

23 Simma B, Frritz $M$, Fink $C$, et al. Conventional ventilation versus highfrequency oscillation: hemodynamic effects in newborn babies. Crit Care Med 2000;28:282-4.

24 Gournay V, Cambonie G, Roze JC. Doppler echocardiographic assessment of pulmonary blood flow in healthy newborns. Acta Paediatr 1998;87:419-23.

25 Mandelbaum-Isken VH, Linderkamp O. Cardiac output by pulsed Doppler in neonates using the apical window. Pediatr Cardiol 1991;12:13-16.

26 Maroto $E$, Fouron JC, Ake E, et al. Closure of the ductus arteriosus: determinant factor in the appearance of transient peripheral pulmonary stenosis of the neonate. J Pediatr 1991;119:955-9.

27 Phillipos EZ, Robertson MA, Byrne PJ. Serial assessment of ductus arteriosus hemodynamics in hyaline membrane disease. Pediatrics 1996;98: 1149-53

28 Kluckow M, Evans N. Low superior vena cava flow and intraventricular haemorrhage in preterm infants. Arch Dis Child Fetal Neonatal Ed 2000;82:F188-94.

29 Evans N, lyer $P$. Incompetence of the foramen ovale in preterm infants supported by mechanical ventilation. J Pediatr 1994;125:786-92.

30 Johnson GL, Breart GL, Gewitz MH, et al. Echocardiographic characteristics of premature infants with patent ductus arteriosus. Pediatrics 1983;72:864-71 